\title{
Practicum en re-construcción: Tejiendo colectivamente un modelo de prácticas desde la innovación
}

\author{
Practicum in reconstruction: Collectively weaving a pattern of practices from \\ innovation
}

Ana M. Novella Cámara

Universitat de Barcelona, España

\section{Resumen}

Los proyectos forman la identidad de las personas, y las personas construyen la identidad de los proyectos. El objetivo de este artículo, es describir la trayectoria del practicum dentro de los estudios de educación social de la Universidad de Barcelona a partir de identificar los elementos que han impulsado su construcción y reconstrucción. La historia de este practicum se caracteriza por las diferentes decisiones que se han tomado, y los permanentes cuestionamientos de éstas, en los casi veinte años de funcionamiento. Pero, no sólo las decisiones tomadas han supuesto un avance o evolución sino también la forma en que éstas se han tomado. Esta evolución va de la mano de tres planes de estudios que han supuesto la oportunidad de revisar y reflexionar qué era el practicum y qué debería llegar a ser. El presente, pasado y futuro del practicum ha sido un proceso de reconstrucción desde la reflexión y evaluación de los agentes implicados. No ha sido una trayectoria en solitario, sino que ha supuesto un proceso colectivo que ha configurado los diferentes espacios formativos y la función docente de los agentes implicados. El camino que hemos andado, y trazado, nos ha permitido evolucionar en los planteamientos, aprender de la experiencia compartida y construir nuestra identidad. Esta evolución permite configurar las prácticas dentro del Grado de Educación Social a partir del modelo de partenariado de reciprocidad, modelo que se ha de consolidar y sistematizar para seguir innovando colectivamente.

Palabras clave: Practicum, estudiante, tutor-universidad, tutor-centro, modelo de partenariado de reciprocidad, Educación social. 


\begin{abstract}
The projects form the identity of the people, and the people define the identity of the projects. The aim of this article, is to describe the trajectory of the practicum within the studies of social education of the University of Barcelona from by identifying the elements that have stimulated their construction and reconstruction. The history of this practicum is characterized by the different decisions that have been taken, and the permanent questionings persistent questions that remain, in the nearly twenty years of operation. But, not only have the decisions made assumed an advanced or evolution of the practicum, but also the way in which these decisions have been made. The evolution has been through three currila which gave the opportunity to review and reflect what the practicum was, and what we want the practicum to be. The present, past and future of the practicum has been a process of reconstruction from the reflection and evaluation of the implied agents stakeholders. It has not been a solo career, but it has been a collective process that set the different training areas and the educational function from the stakeholders. The way we have went, and layout, has allowed us to evolve approaches, learning from shared experience and build our identity. This evolution can set practices within the Social Education Degree from a reciprocal partnership model, a model that has to be consolidated and to systematized to continue to innovate collectively.
\end{abstract}

Key words: practicum, student, university tutor, center tutor, reciprocal partnership model, Social Education

\title{
Introducción
}

La trayectoria del practicum en los Estudios de Educación Social se remonta al año 1993. Momento en que en el marco de la Facultad de Pedagogía nacieron estos estudios, desde entonces hasta ahora son tres los planes de estudios que se han renovado que han potenciado períodos de reflexión encaminados a dar pequeños pasos que nos aproximasen hacia la mejora de la formación práctica.

El objetivo de este artículo es exponer los diferentes avances del practicum que han supuesto innovaciones y transformaciones en los planteamientos organizativos y formativos. Estas transformaciones han configurado la identidad de la propuesta formativa de las prácticas externas que se desarrollará el próximo curso académico (2011-2012). El contenido que aquí se expone es una construcción colectiva, fruto del trabajo de las diferentes coordinaciones de practicum que desde 1993 han velado por la calidad de las prácticas; de las aportaciones de los diferentes profesionales que en este período se han implicado como tutores-centro y en la avaluación de estos procesos; de las experiencias personales de los estudiantes que han cursado los estudios y que han reflexionado sobre su trayectoria formativa en la práctica; por el compromiso de los diferentes equipos docentes que curso tras curso han velado por mejorar su docencia y por construir una propuesta docente colectiva; por las incansables reivindicaciones de las diferentes jefaturas de estudios y de los diferentes equipos de decanato que han apostado por un practicum de calidad a pesar de las dificultades y limitaciones institucionales. Este practicum, nuestro practicum, se explica por su pasado, por su presente y su proyección de futuro. Es el que es gracias a la huella que han dejado año tras años los diferentes agentes implicados que han compartido el reto de reconstruir un practicum desde el presente para el futuro.

A continuación, se analiza como ha evolucionado los planteamientos del practicum a lo largo de la historia de los estudios. 


\section{Hilvanando las primeras condiciones}

En 1996, en la evaluación institucional que se hizo de los estudios de Educación Social se evidenció ciertas carencias en la asignatura del Practicum, que se concretaban en determinadas dificultades en la gestión de las prácticas, sobretodo por el creciente volumen de estudiantes y por la poca consideración que tenía esta docencia para el profesorado. En consecuencia, se puso de manifiesto la necesidad de reforzar la gestión administrativa para poder alcanzar una mejor eficacia. Un reforzamiento que implicaba cuestionarse la constitución del equipo de coordinación dedicado a las prácticas y las funciones que había de desarrollar. En definitiva, todo acabaría revirtiendo en una mejor calidad de las prácticas tanto a nivel de docencia como de coordinación con los centros de prácticas.

Esta evaluación fue la antesala de la primera reforma de plan de estudios del 1993, que no se comenzó a implementar hasta el curso 2001-2002.

El resultado de la evaluación institucional del practicum de Educación Social llevó a tomar dos decisiones clave. Éstas han incidido en la trayectoria del practicum marcando un punto de inflexión en el que se impulso una revisión en profundidad.

Dotar a la coordinación de más recursos humanos y restringir la función administrativa. El equipo de coordinación desde ese momento paso de estar constituida por tres integrantes a constituirse por seis integrantes, dos representantes de cada departamento. Este cambio permitió que la coordinación no sólo cumpliese funciones organizativas sino que velase por el desarrollo del practicum dentro del nuevo Plan de estudios. Se analizó la necesidad de liberarla de tareas administrativas para centrarse en otras funciones más relevantes.

Formar parte del Plan Piloto de las titulaciones adaptadas al Espacio Europero de Educación Superior (EEES). El consejo de Estudios de Educación social propuso ser una titulación que iniciaría el proceso de adaptar los Planes docentes al sistema de créditos europeos en el curso 2004-2005, en aquel momento los desconocidos European Credits Transfer System (ECTS). Esta decisión, coincidió con la responsabilidad que asumió la coordinación de la revisión del practicum dentro del nuevo plan de estudios. La Coordinación del Practicum, consideró oportuno empezar a trabajar en esta doble línea, en cierto modo coincidía con la concepción que teníamos de integrar diferentes formas de formación teórica y práctica dentro y fuera del aula universitaria, y de cómo el estudiantado debería trabajar para lograr los objetivos educativos.

La repercusión de estas decisiones ha configurado aquello que define y caracteriza a la Coordinación de Practicum que desde sus orígenes ha sido constituido por un equipo interdepartamental y por lo tanto interdisciplinar.

La coordinación del practicum de Educación Social es el órgano encargado, por delegación de la Jefatura de Estudios y la Facultad de Pedagogía, de velar por la calidad del practicum. El concepto de calidad también ha cambiado y evolucionado en este tiempo. Atrás ha quedado el reduccionismo de calidad vinculada a garantizar plazas de prácticas para todos los estudiantes, para dar un paso y visualizar su responsabilidad en la búsqueda y seguimiento con los centros de prácticas colaboradores. Para 
posteriormente dar otro paso, reconociendo a este órgano la función de acompañar en la elaboración de los planes docentes de las prácticas externas, impulsar la función docente de los centros de prácticas, garantizar la idoneidad de la oferta de plazas para la formación en competencias profesionales, velar por los procesos de mejora como asignatura, entre otras funciones. Además, algunas de las integrantes de la coordinación del practicum también asumen créditos de esta asignatura, así experimentan en primera línea las implicaciones de las decisiones tomadas y la evaluación continuada de su proceso y resultados.

La metodología de trabajo de la coordinación es de carácter horizontal, reflexivo y deliberativo. Las decisiones se toman desde la investigación evaluativa y en concreto desde la investigación-acción como proceso de permanente revisión de aquello que se ha decidido e implementado, para volver a planificar el siguiente paso. La coordinación, revisa permanentemente las decisiones que toma y a partir de la avaluación acuerda mejoras para ediciones posteriores. En reuniones de carácter semanal, el equipo trata los diferentes temas que van surgiendo pero también trabaja con una perspectiva de futuro intentando desprenderse de la inmediatez y urgencia. Entender su metodología de trabajo como investigación evaluativa ha favorecido implicarse en proyectos de investigación. Éstos han posibilitado profundizar en las necesidades identificadas con la voluntad de proponer mejoras fundamentadas desde las percepciones de los participantes. Todos los proyectos de investigación o de innovación docente han aportado evidencias para la transformación. Algunos de estos proyectos son:

"La evaluación y selección de centros de prácticas de educación social" (2001, 10/V/AD-A1/07/FREI);

Diseño y elaboración de la guía "El Practicum als estudis d'educació social: pla formatiu"(2005);

Proyecto de transversalización de los estudios de educación social (2006PID-UB/08).

"Análisis y avaluación de la transferibilidad de competencias profesionales de la Educación Social en los centros de prácticas" (2008MQD155).

“La función docente en las prácticas externas. Investigación de un diseño curricular corresponsable entre los Estudios de Educació Social y el mundo laboral."(REDICE-10/1002-27);

"Validación de los Quadernos formativos de las prácticas: La transferencia de las competencias profesionalizadoras en Educación social" (2010PID-UB/76)

La coordinación no es un oasis en medio de un desierto, sino que está en permanente contacto con los agentes implicados. No es un espacio aislado que toma las decisiones en solitario sobre el modelo de practicum, sino que esta en red con los implicados y en coordinación con ellos toma decisiones y revisa los resultados de los procesos diseñados para el avance. $Y$ como veremos más adelante ha ido diseñando diferentes espacios para fomentar esta corresponsabilidad. 
Esta concepción, de qué es y cómo trabaja la coordinación, ha permito en este período dar pasos hasta alcanzar lo que sería un modelo de practicum propio fundamentado en unos principios fundamentales que le dan sentido y le organizan en estructuras de acción.

\section{Tejiendo el sentido y significado de las prácticas}

La definición de las prácticas externas comporta una perspectiva diferente de formación. Según Zabalza (2011), encontramos cuatro tipos de practicum:

1. Orientado a la aplicación de aquello aprendido.

2. Orientado a facilitar el acceso al trabajo

3. Orientado a completar la formación general con otra más especializada.

4. Orientado a enriquecer la formación básica completando los aprendizajes académicos con la experiencia a los centros profesionales.

Es desde esta última orientación que se plantean las prácticas externas en el Grado de Educación Social. Las prácticas han pasado de ser una asignatura más en la que hacer horas en un centro a ser el eje central del plan de estudios. Ser eje posibilita que otras asignaturas organicen sus contenidos teniendo la formación práctica como referencia e impulsen la transferencia de competencias a través de la reflexión, teorización y sistematización de aprendizajes teórico-prácticos. Llegando a impulsar trabajo transversal entre asignaturas que fomentan las competencias específicas y transversales del profesional. Así, las prácticas externas son el espacio central de significación y de encuentro de la formación a la universidad y la formación a los centros de prácticas. En ambas experiencias formativas, en los dos contextos de aprendizaje, se inscriben tanto elementos teóricos como prácticos. Estos dos contextos han de mantener una relación simétrica que se deberá basar en un modelo de partenariado (Zabalza, 2011).

El recorrido para llegar a un modelo de partenariado interinstitucional, nos ha llevado a identificar diferentes modelos en nuestra trayectoria hasta llegar un modelo acorde a la filosofía de los ECTS. Todos ellos se diferencian por la relación que se establece entre los agentes implicados y el papel que tiene cada uno de ellos hacia los otros.

Un primer modelo, muy arcaico pero que existió, se caracterizaba por un carácter individualista y una estructura unidireccional con unidades formativas aisladas. Este modelo de practicum, como se puede ver en la figura, comportaba que los miembros implicados desarrollaban su acción de forma aislada sin conocer lo que pasaba en las otras partes implicadas. Los estudiantes recibían de la Universidad orientaciones teóricas y del Centro los referentes prácticos, dejando en él la tarea de poner en relación estos conocimientos. Universidad y centro preparaban sus escenarios para que el estudiante desarrollara su formación práctica, pero no interrelacionaban ni se visualizaban desde la formación compartida. 


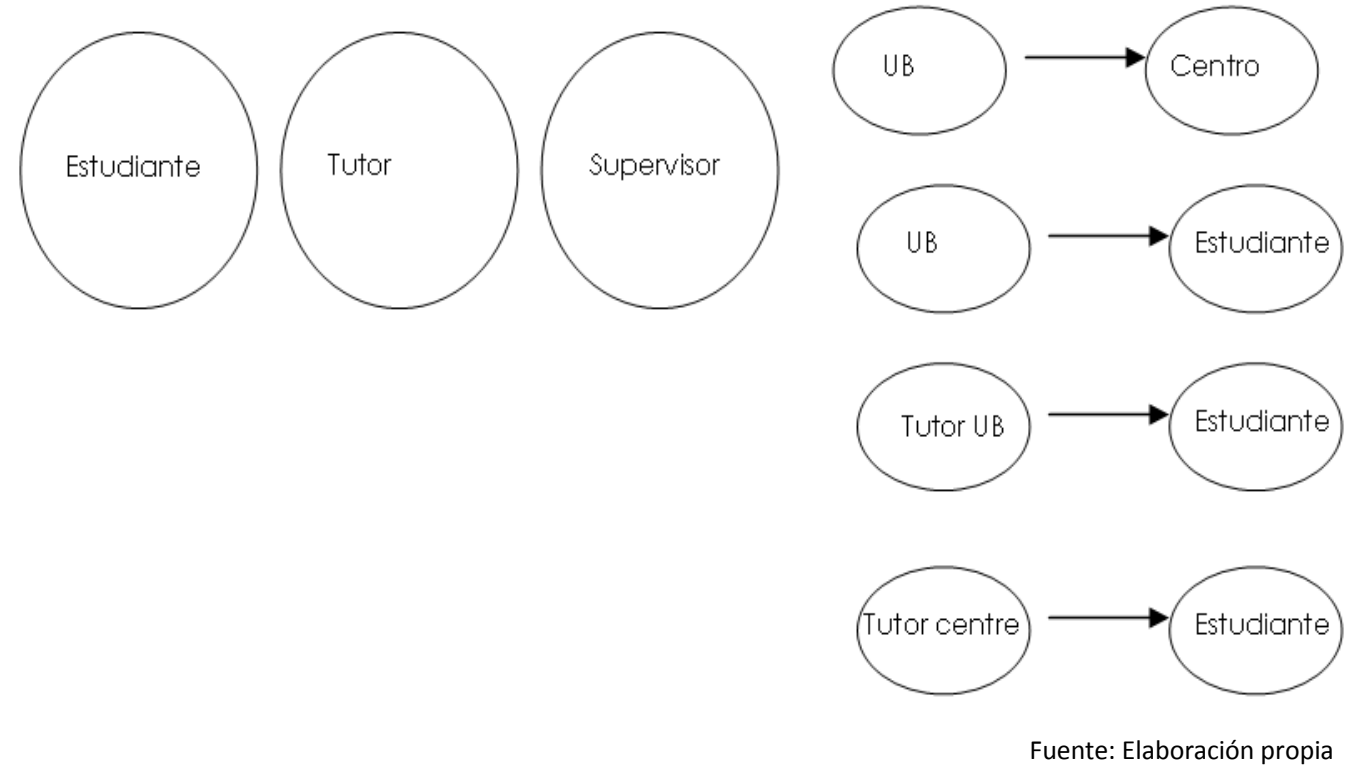

Figura n.1. Modelo 1 "Unidireccionalidad e individualista".

Otro modelo de formación en el practicum, presente en los inicios con la voluntad de superar el modelo anterior, se caracterizaba por la voluntad de poner en relación a las partes implicadas y por organizar unos protocolos que favoreciesen el acompañamiento que el centro y la universidad daban al estudiante. En este modelo, los elementos que intervienen en el practicum, a diferencia del anterior, reciben una influencia mutua en su intervención. Tutor y supervisor se tienen en cuenta a la hora de organizar el espacio de formación, y tienen en cuenta las acciones que el otro tiene sobre el estudiante. Entienden que la formación tiene lugar tanto en el centro profesional como en la Universidad. Aún así, el estudiante continúa siendo un mero receptor de aprendizajes de uno y de otro agente sin tomar parte activa.

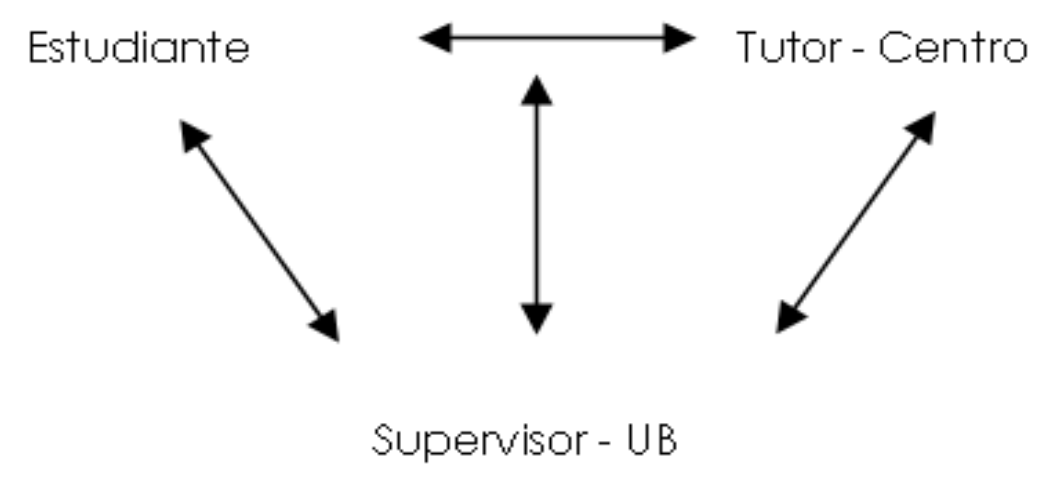

Fuente: Elaboración propia

Figura n.2.Modelo 2 "Bidireccional" 
La evolución de este último modelo, nos nutrió de las teorías sistémicas, en las cuales aquello que influye en el comportamiento de las personas tenía que ver a la relación que se establecía en todos los elementos que configuraban el sistema. Así, en un tercer modelo se pretendía rectificar la bidireccionalidad para reconocer la intersección de los procesos y aprendizajes que acontecían en cada escenario formativo. Así como, reconocer que en el proceso formativo intervienen otros aspectos, que hasta ahora no se habían tenido en cuenta (motivaciones, emociones, experiencias previas...), aun cuando intervenían en la relación educativa que se daba en el espacio de las prácticas. Cada uno de los agentes que intervienen en las prácticas traía, a este contexto, referentes teóricos y prácticos que han adquirido en otros escenarios y hacen que este bagaje tanto personal como académico ofrecía referentes en la formación (que no se empezará de cero).

Estos contextos en qué participan los agentes entran en interrelación y se nutren mutuamente, teniéndose presentes para organizar y orientar los espacios formativos.

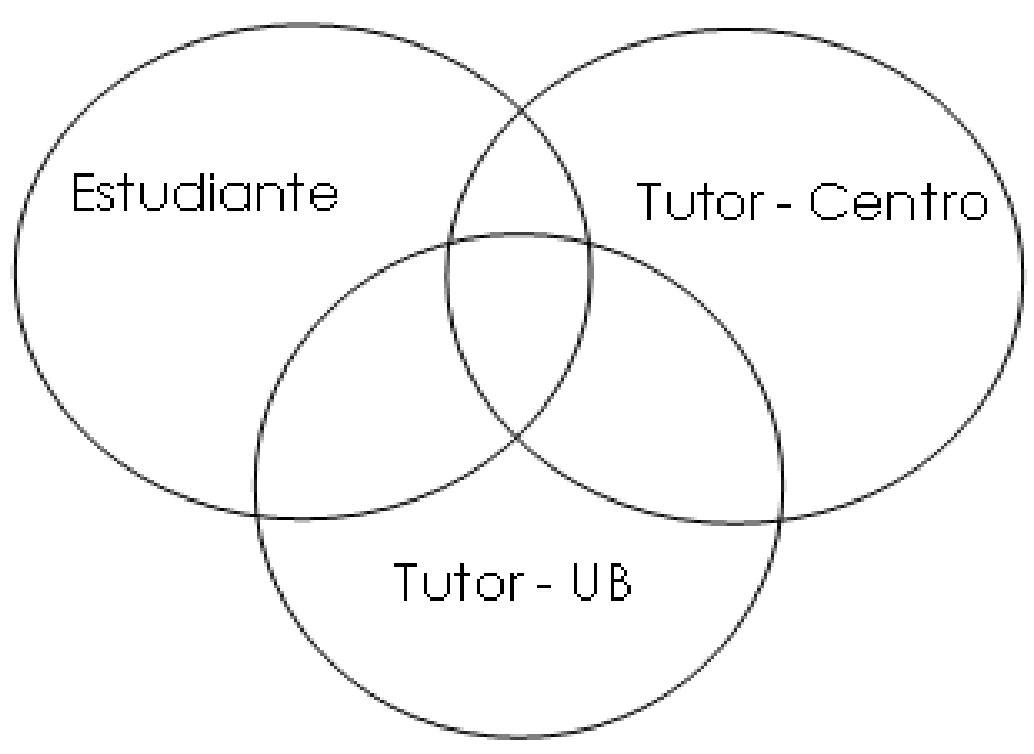

Fuente: Elaboración propia

Figura n.3.Modelo 3 "Intersección y yuxtaposición"

En el momento que pasamos a ser una de las titulaciones piloto que iniciábamos la aventura de los EEES, quisimos desprendernos de estos tres modelos para definir otro desde el que poder reconocer la implicación de todos los agentes y algunas de las variables contextuales que incidían en la formación del practicum. La presentación estructural del modelo, fue diseñado para difundir entre todos los agentes implicados cual era su rol dentro de la estructura formativa del practicum. Brevemente, diremos que se caracteriza por dar el protagonismo del proceso al estudiante, quien en último término es el responsable del aprendizaje. Aprendizaje que no hace en solitario, sino que se da siempre en un contexto social, donde los responsables de la función docente (tutor y supervisores) tienen una propuesta curricular de aquellos mínimos que el 
estudiante como futuro titulado debería lograr y hay una propuesta de contenidos del que se considera que ha de adquirir. No sé si podríamos decir que en este modelo hay un imaginarium, más o menos compartido, del perfil de profesional que queremos formar en el marco de la Diplomatura de Educación Social de la Universidad de Barcelona.

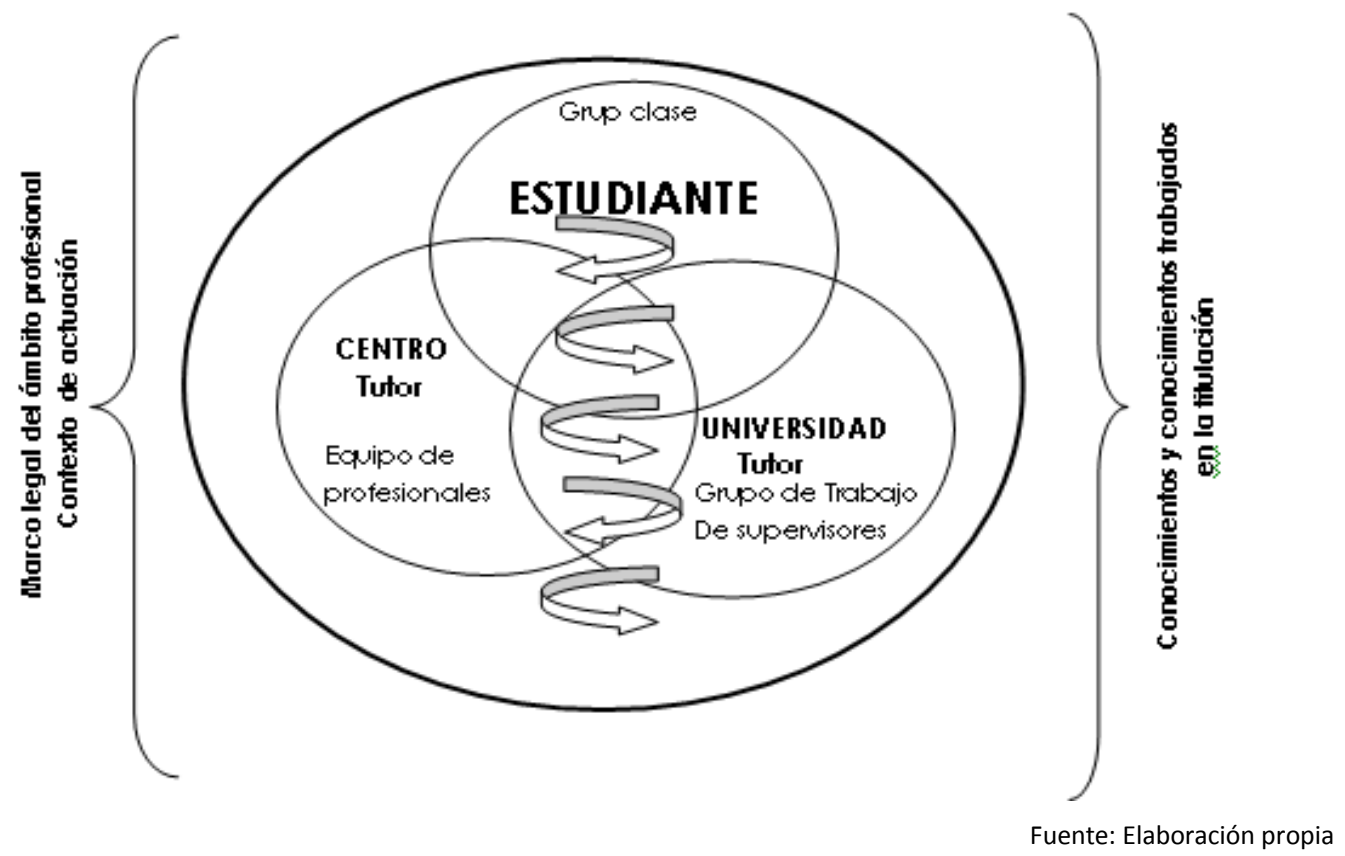

Figura n.4.Modelo 4 "Centrado en el estudiante"

Otro elemento significativo de este modelo, y diferenciado de los anteriores, es que se entiende el proceso formativo del practicum como un proceso participativo, reflexivo y dialógico. En él, se pretende que el estudiante construya el marco de referencia de su práctica, que, a partir de elementos teórico-prácticos, vaya analizando, interpretando y estableciendo referentes, con la finalidad de consolidar contenidos y experiencias que surgen en la práctica profesional. En este modelo, los contenidos de trabajo, recogidos en una propuesta curricular, tienen su origen en la acción del estudiante. Acción que se da tanto en el espacio de las prácticas del centro como en el espacio "académico" de la Universidad. El proceso de interiorización que hace el estudiante es resultado de, como dice Zabalza, diferentes espacios de conversación donde uno va generando su propio discurso en relación al discurso de los otros. El conocimiento, aun cuando se mantiene como una adquisición personal, va surgiendo de las aportaciones de los otros y del contraste entre las propias ideas y la de los otros. Aprendemos en un marco cultural, en el seno de las instituciones, en relación a los intercambios con los otros. 
Por último, y dentro de lo que sería el modelo de practicum referencial en el marco del desarrollo del Grado de Educación social, el último modelo "el partenariado de reciprocidad o de corresponsabilidad" es la evolución del cuarto modelo, a partir de la consolidación del trabajo por competencias. La búsqueda y las propuestas de mejora llevadas a término en el practicum han llevado a reconsiderar la relación entre los agentes (estudiantes, Tutor-universitario y tutor-centro) las funciones de los cuales se concretan y se comparten en una relación simétrica y de igualdad sobre una base de corresponsabilidad en la formación. Esto significa reconocer que cada agente tiene una experiencia a compartir, un saber, un saber ser y un saber hacer. Los estudiantes no quedan excluidos pues son un agente más de las prácticas y el protagonista de su aprendizaje. Estos tres agentes principales, a nivel micro unidad, se constituyen como equipo formativo o equipo docente con funciones compartidas pero también específicas.

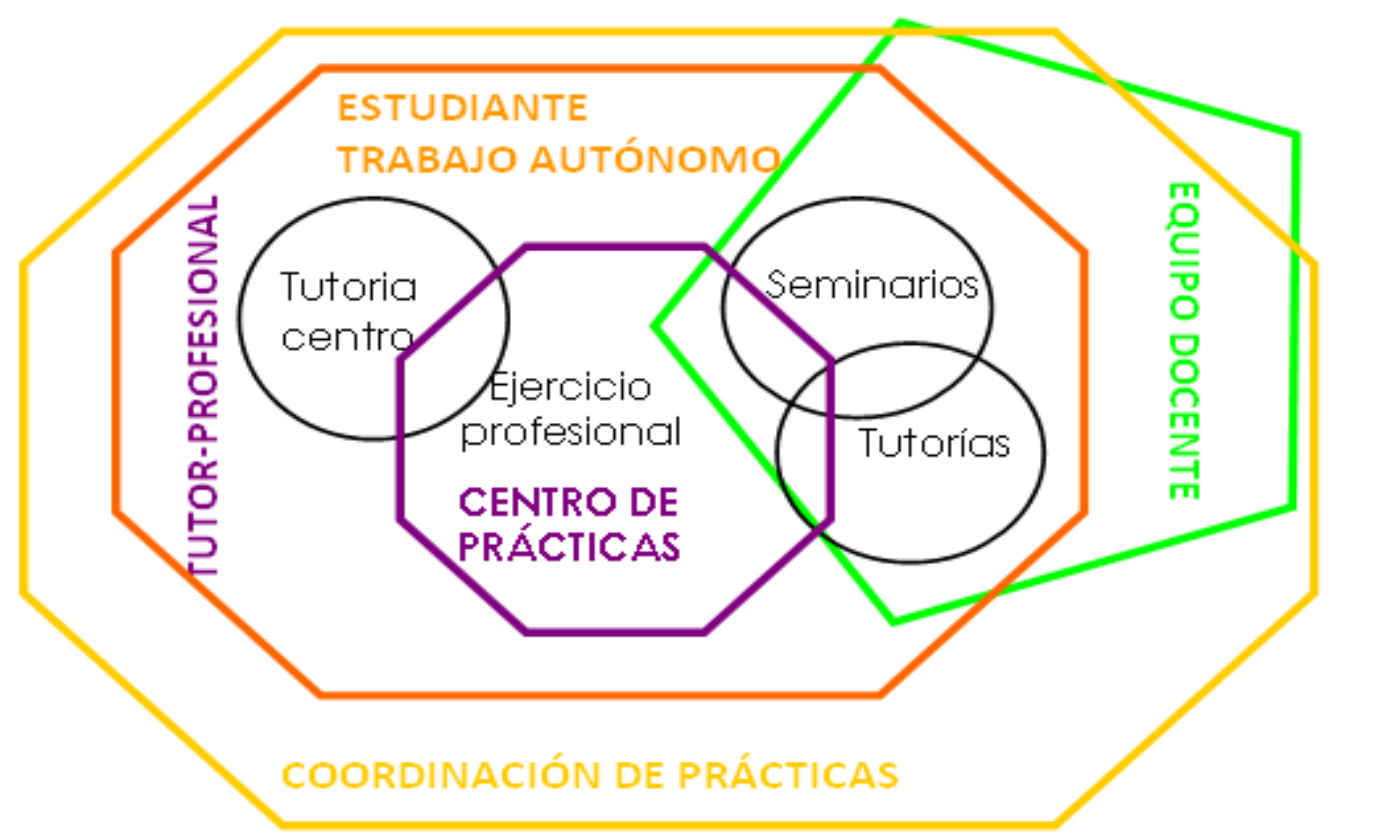

Fuente: Elaboración propia

Figura n.5. Modelo 5 "Partenariado de reciprocidad o de corresponsabilidad"

Desde una formación por competencias, el mundo universitario y el mundo laboral se convierten en centros de formación. Los estudiantes son el eje central en la configuración del proceso de enseñanza-aprendizaje. Los tutores de centro, los tutores universitarios y el estudiantado son corresponsales de la formación práctica.

A partir de los referentes señalados en este modelo, entenderemos el Practicum como un contexto formativo dónde el proceso de enseñanza-aprendizaje -lleno de encuentros, conocimientos, aprendizajes y vivencias- se convierte (no solamente) en aquello que debe garantizar una formación global respeto los saberes y conocimientos más académicos, sino también en una formación integral que prepare al estudiante para la vida profesional y, también, porque no decirlo, para el desarrollo personal. 
Este modelo se ha tejido a partir del proyecto de investigación "Análisis y avaluación de la transferibilidad de competencias profesionales de la Educación Social en los centros de prácticas" (2008MQD155). Diferentes grupos deliberativos de tutores-centro, estudiantes y tutores-universitarios discutieron sobre como se representaban la transferencia de competencias y proceso de las prácticas. A partir de estos resultados, se han elaborado tres cuadernos guía que tienen como finalidad ser una herramienta de consulta y apoyo para los profesionales tutores, estudiantes y docentes universitarios para potenciar la transferencia de competencias del estudiante. $Y$ se propone, como curriculum oculto, potenciar el partenariado de reciprocidad.

\section{Tejiendo colectivamente más allá de la coordinación}

Acercarse a un modelo de practicum fundamentado en el partenariado de reprocidad, o la más conocida corresponsabilidad, no es tarea fácil. Los pasos que se han dado se han aproximado a ello muy lentamente sin estar muy convencidas de haberlo alcanzado. El otro día, Eduardo Galeano definía la utopía como aquello que nos sirve para caminar. Caminar en el practicum nos permite perseguir una máxima probablemente inalcanzable pero que hace que continuemos caminando.

La coordinación no podía ni quería caminar en solitario. La coordinación no ha sido nunca una estructura vertical sino que su funcionamiento se caracteriza por la horizontalidad y participación de los diferentes agentes implicados. Principalmente, tutores-centros y tutores-universitarios que son agentes que mantienen estable su relación y quienes han de diseñar su escenario y estrategias docentes en beneficio de la formación del estudiante para incidir en la transferencia de sus competencias año tras año. Los estudiantes participan siendo consultados en espacios de análisis y discusión, las evaluaciones y otros espacios informales más personales y próximos.

Los diferentes equipos docentes implicados en las asignaturas del practicum han sido grandes cómplices de los avances en los diferentes procesos de implantación de esta asignatura dentro de los tres planes de estudios. Sin ellos, no hubiese sido posible caminar. Los diferentes equipos docentes, desde la cotidianidad de la docencia en el practicum, ha constituido un espacio reflexivo y constructivo alrededor de los ejes más importantes de la función docente revisando y consensuando objetivos, contenidos, metodología y evaluación. En cada uno de estos procesos, se ha caminado hacia un proyecto colectivo, un proyecto formativo conjunto ante el reto de superar el anterior y sistematizar las buenas prácticas docentes que toman y dan sentido en la relación educativa con el estudiante y con el análisis de su experiencia práctica. Pero no sólo, hemos avanzado juntos en aspectos de plan docente sino también en otros aspectos que potenciasen la interrelación y corresponsabilidad entre los agentes. Inicialmente desde la preocupación de facilitar la comunicación entre agentes, el seguimiento de la función docente del tutor, el establecimiento del plan de trabajo y en la actualidad desde el hacer juntos, desde encontrarnos en la corresponsabilidad de potenciar la transferencia de las competencias con los estudiantes.

Ha sido un espacio de enriquecimiento mutuo dónde ha sido posible aprender y construir una didáctica del practicum. Ha sido un espacio que nos ha llevado a 
establecer unos criterios mínimos para esta docencia, pero que se han convertido en máximos del modelo que hemos experimentado. Ahora bien, todo y el trabajo que se ha hecho sobre la propia práctica docente, hace falta decir que los estudiantes continúan señalándonos que debemos coordinarnos para establecer unos criterios comunes para todos y todas.

Los espacios de encuentro con los tutores-centros colectivos e individuales han sido otro gran avance. La creación de estos espacios de encuentro surgió de la necesidad de reforzar y consolidar espacios y canales de comunicación y relación con los tutores-centro desde la coordinación y los tutores-universitarios. Entendíamos que favorecer el encuentro entre los agentes, potenciaría el acompañamiento y el intercambio sobre el proceso de enseñanza y aprendizaje, hecho que repercutiría en una mejor calidad de la formación en el espacio de prácticas. En relación a este aspecto, se han ido introduciendo ciertas mejoras que han ritualizado y sistematizado estos espacios siendo permanentes y formando parte de la relación educativa.

La coordinación tiene mucha relación con los responsables de los centros para establecer las plazas, hacer un seguimiento y avaluar como ha ido para poder volver a un curso más. Pero se reconoció, que con los tutores-centro no, y se veía como éstos tenían poca información de las condiciones del practicum y del proceso. Hecho que dificultaba la relación con el estudiante y con la universidad. Inicialmente se pensó, que aumentando el número de visitas al centro por parte del tutor-universidad sería suficiente. De ahí que se propuso mantener tres encuentros entre supervisor y tutor, en las que también debería participar el estudiante. Pero, cuando se es tutoruniversitario una se da cuenta que es prácticamente imposible dado que supervisa a unos quince estudiantes. Por lo tanto, siendo conscientes de esta limitación reducimos a una la visita al centro, dejando para los casos excepcionales una segunda visita. Sin perder de vista la necesidad de trabajar por la corresponsabilidad, las vistas al centro evolucionaron hasta institucionalizar dos encuentros en la universidad.

Un primer encuentro, a principios de curso, que tenía la finalidad de presentar el modelo de practicum a los tutores del centro y poner en relación a tutor-centro con tutor-universitario. La coordinación de practicum considero que tenía la responsabilidad de compartir con los tutores-centro y tutores-universitarios las directrices del practicum tanto a nivel conceptual como a nivel organizativo, aprovechando para introducir las novedades de cada edición. En un segundo momento del encuentro, por grupos de supervisión se encontraba el tutor-universitario con todos los tutores-centro con los que trabajaría durante el curso y con quienes compartiría la especificidad de su ámbito y de la docencia. En las tres últimas ediciones, que nos han ayudado a caminar hacia el practicum del grado de educación social, el encuentro tenía un tercer momento en el que se incorporaba el grupo de estudiantes que supervisaría el tutor-universitario y el tutor-centro respectivamente. En este espacio, de unos noventa minutos, el tutor-universitario dinamizaba un seminario donde principalmente los tres agentes consensuarían que entienden por el practicum, por la formación del educador social y compartirían el papel del educador social en el territorio que trabajan. La valoración de este encuentro es muy positivo para todos los implicados, pues no sólo tiene una finalidad informativa sino que la formación que genera satisface a todos y ayuda a aproximarse unos a los otros 
generando un conocimiento mutuo fundamental para la relación de corresponsabilidad.

Un segundo encuentro, a finales de curso, que se ha consolidado y porque no decirlo se ha institucionalizado como las Jornadas del Practicum. Se han cerrado los estudios de la diplomatura celebrando la novena edición de estas jornadas donde participan tutor-centro, estudiante, tutor-universidad y coordinación. A diferencia del primer encuentro, éste tiene la finalidad de celebración, agradecimiento y reconocimiento del trabajo compartido. Por ello, se propone una conferencia alrededor de un tema de interés para todos y todas. Un espacio de café, centrado para el intercambio y "despedida" donde los tutores-centro y tutores-universitarios aprovechan para valorar la evolución del estudiante y para cerrar el proceso; y donde se acostumbra a incorporar el estudiante. A partir de aquí, hemos ido reformulando el sentido del cierre. En las primeras ediciones, poníamos el acento en aprovechar el encuentro de todos los agentes para analizar y debatir aspectos concretos de la evolución del practicum. Era un espacio para la evaluación conjunta del modelo e identificar las transformaciones que se requerían y sobre las que la coordinación trabajaría para superarlas. En las últimas cuatro ediciones, transformamos este momento en un espacio para compartir experiencias de prácticas significativas y relevantes. Experiencias que podían ser trabajos de prácticas que se habían diferenciado por alguna cuestión específica y que eran presentados por estudiante y tutor-centro o tutor-universitario; y experiencias más personales y de carácter valorativo que un grupo de estudiantes habían compartido y querían extender a sus compañeros. Esta innovación permitía a todos expresarse e identificar elementos evaluativos desde experiencias y realidades de las prácticas concretas. El giro centrándonos más en el estudiante satisface más a todos los participantes y permite reconocerse en los aprendizajes alcanzados.

Los espacios de encuentro han sido centrales para caminar juntos y tejer la corresponsabilidad formativa. Hemos alcanzado más proximidad, más conocimiento mutuo y compartido en el plan formativo que nos proponemos. Pero sobretodo, se ha construido una forma de entender el practicum y las prácticas del estudiante identificando tres espacios de aprendizaje que se dan dentro del centro de prácticas, de la universidad (seminarios y tutorías) y del trabajo personal del estudiante. Toledano (2004) propone dentro del practicum, como contexto y proceso formativo, tres espacios de aprendizaje que pese a resultar difícil y arriesgado delimitar las fronteras que los definen serian:

Espacio de ensayo.El practicum como espacio de ensayo es el lugar dónde el estudiante toma contacto, conoce y vivencia a partir de la observación y participación de la vida, dinámica y propia práctica cotidiana, integrando de alguna manera ciertas habilidades, estilos y actitudes presentes en la realidad profesional. Esta aproximación queda garantizada por el acompañamiento y guia del referente profesional que crea entornos de seguridad y de cesión de responsabilidad para que el estudiante ejercite y ensaye progresivamente la diversidad de actuaciones, estrategias y técnicas que son presentes en las intervenciones socio-educativas.

Este espacio debe permitir que los contenidos trabajados en la formación estrictamente académica puedan ser transferidos a la prácica, siendo resignificados y reconstruidos al ser interpelados por la experiencia práctica. Y esto, por medio de un proceso de confrontación, redefinición y comprensión que posibilite que los diferentes 
conocimientos conceptuales, técnicos y éticos puedan ser elaborados, cuestionados y ratificados y, así, se integren de tal forma que acontezcan aprendizajes significativos.

Espacio de inmersión. No se puede negar que es en las prácticas donde el estudiante hace una immersión en la realidad y práctica profesional de forma contextualizada. El practicum se convierte en el movimiento principal de toma de contacto con el mundo profesional, donde se van conociendo e identificando las funciones y competencias profesionales. El estudiante a partir de este contacto en "primera persona" con la realidad profesional, puede conocer y ejercitar (y poner a prueba) su vocación e identidad profesional.

La convivencia regular y estable permite, sin duda, al estudiante disfrutar de una visión profunda de la profesión y, así, ir construyendo en su imaginario aquello de real y de ficticio que, de manera predeterminada, tenía respeto la profesión. Esto sólo es posible con un trabajo desde este espacio de inmersión de la práctica. Por todo esto es importante que el practicum, entendido como espacio de inmersión, posibilite el ejercicio de aprendizaje profesional mediante el ejercicio de la profesión y con la ayuda de un profesional que no solamente actúa como tal sino que asume la función docente. Esta función consiste en acompañar el proceso del estudiante, permitiendo en este "ejercicio" acercarse a la profesión, la vivencia y el contacto con los problemas y la realidad profesional.

Espacio vivencial y de autoconocimiento. Este espacio de la práctica supone la parte vivencial de la formación que permite un trabajo de reflexión y comprensión significativa respeto la relación y acción socioeducativa y profesional en los entornos donde se da. Y también, respeto a uno mismo dado que el estudiante, a partir de la aproximación progresiva a la realidad con sus retos y dificultades, va poniendo en juego sus competencias y propias maneras de hacer, pero observando, aun así, las maneras y estilos profesionales presentes en el recurso de prácticas, de tal manera que las va analizando, probando y progresivamente integrando dentro de su propio repertorio de respuestas.

La experiencia de sentirse puntualmente y temporalmente cono un profesional es el que, de alguna forma, permite al estudiante responsabilizarse, elaborar y tomar conciencia del sentido y complejidad de la tarea y práctica profesional. Para el estudiante representa un trabajo introspectivo, de autoconocimiento y de identificación respeto sus competencias así como de las propias dificultades, limitaciones y, incluso, miedos que le han de obligar a escucharse y sincerarse consigo mismo para descubrir y/o ratificar sus motivaciones personales y profesionales. Pero sobretodo para construir de forma activa y consciente su identidad como profesional de la educación social. Desde este espacio vivencial del practicum, dónde inevitablemente entran en juego diversidad de sensaciones, sentimientos, contradicciones y pequeñas o grandes frustraciones, el estudiante desarrolla un trabajo personal de clarificación, reflexión y análisis de la propia vivencia para comprenderla e integrarla. De esta forma poder elaborar de forma crítica y reflexiva los diferentes retos que supone el aprendizaje de la práctica profesional. Este proceso supone la puesta en escena, en la cual el estudiante representa el rol para el que se está formando. Un espacio, a partir del que, el estudiante se ve, se vive y se autoanaliza.

Todos estos espacios formativos y los diferentes aprendizajes de la formación práctica que de ellos emergen, son contenidos de trabajo no sólo del estudiante, sino sobretodo del tutor-universidad y del tutor-profesional. Éstos son los agentes responsables, y corresponsables, de provocar intencionalmente la reflexión y 
teorización de, desde y para la acción. Son los responsables de significar la práctica desde la teoría y la teoría desde la práctica. Compartir los referentes para contribuir desde los diferentes espacios a la transferencia de competencias y a la construcción/reconstrucción permanente de la identidad es un proceso largo que se ha tejido desde la proximidad y cuestionamiento del proyecto formativo que tejemos juntos desde espacios diferenciados que comparten un mismo objetivo.

\section{Tejiendo un espacio para la construcción de la identidad profesional del estudiante}

Otra evolución significativa en el practicum fue la introducción y sistematización del seminario. Ante el desconcierto del estudiante de no tener los referentes del practicum ni conceptuales ni estructurales, dejados en un tiempo al azar y a la espontaneidad del devenir, se propuso un espacio estable para supervisar al estudiante más allá del que seguirían siendo las tutorías individualizadas. A su vez, los tutores de centro informaban que los estudiantes llegaban con ciertas lagunas conceptuales sobre el ámbito de intervención específico del centro y con cierta desorientación de que hacer en el período de prácticas.

En el Plan de Estudios 1993 revisado, a partir de la evaluación institucional, identificamos la necesidad de organizar el acompañamiento al estudiante y definir un espacio de docencia presencial para garantizar unos mínimos y perseguir algunas máximas. Así, nacieron los seminarios de prácticas.

Los seminarios eran un espacio de encuentro grupal de unos noventa minutos, entre unos quince $\mathrm{y}$ dieciocho estudiantes procedentes de un mismo ámbito mayoritariamente, que serían formados en contenidos propios de éste y en orientados en su paso por el centro. Parte de los contenidos que se trabajan eran los referenciales profesionales pero también eran los experienciales del estudiante, que se intercambiaban en el sí del seminario. Como toda propuesta, en este tiempo ha evolucionado y sistematizado para configurarse como un espacio clave en la formación del estudiante que simulaba el trabajo interprofesional de los equipos de centro. Con la llegada de los EEES, siguiendo las indicaciones situamos al estudiante en el centro del aprendizaje y de la enseñanza. $Y$ en los tres últimos años, a puerta de la implementación del Grado, en el seno del seminario les hemos situado como unos docentes más responsables de su formación y la de sus compañeros. La corresponsabilidad del estudiante se ha impulsado desde los seminarios.

Los seminarios, son un espacio grupal de reflexión teórica sobre la práctica y de diálogo permanente con un mismo, con los otros y con el contexto dónde está inmerso. El tutor/a-universitario debe crear las condiciones para que sea un espacio de aprendizaje, y por lo tanto, un proceso de referencia clave para el estudiante. Éste vive una experiencia muy intensa con el ejercicio profesional en el espacio de las prácticas y es en el espacio de seminario dónde aporta todo aquello que va experimentando y descubriendo, siendo un espacio de ayuda mutua a la vez que un espacio formativo y de aprendizaje. En el seminario, los tutores-universidad acompañan tanto el proceso de inmersión y de observación participativa del estudiante en la realidad social y educativa, como el proceso de creación y aplicación de una propuesta socioeducativa. 
Además, en los seminarios se les acompaña en la transferencia y en el desarrollo de competencias básicas de profesional como por ejemplo: la reflexión, la observación, la crítica, la adaptación a la realidad, la flexibilidad, la interpelación y la interrelación de saberes.

Los espacios de seminarios, por lo tanto, son un auténtico espacio de análisis de la realidad y práctica profesional. Incluye también la introspección del proceso personal y profesional de los estudiantes, y al mismo tiempo, del proceso colectivo de constituirse como grupo de trabajo que permita trabajar aquello que se va adquiriendo, los procesos de aprendizaje, la interpelación y la interrogación constantes, las competencias que se ponen en juego, las nuevas propuestas creaciones y su aplicación.

Los seminarios nunca son iguales y no podemos pretender que lo sean. Cada curso y cada grupo aportan elementos propios y particulares que hacen que la relación educativa sea única e irrepetible; que los contenidos que se construyan son aquellos que el grupo y cada integrante, aportan y tienen sentido por su evolución; que las propuestas educativas y formativas sean las que se deriven del capital humano integrante del grupo;... Los seminarios son un mar de oportunidades y retos que permitirán a cada estudiante aprovechar el que allá emerge a partir de sus necesidades, intereses, bagaje y disposiciones. Por esto, es necesario que cada participante, cada estudiante, decida conscientemente qué grado de implicación y dedicación está dispuesto a tener. Hay unos mínimos establecidos para garantizar el aprendizaje pero los máximos dependerán de cada uno de ellos y ellas. Esta es otra de las particularidades del seminario por el cual tiene un valor especial.

Los objetivos generales de estos seminarios son:

- Ayudar a aclarar/formular/expresar motivaciones, expectativas y objetivos individuales ante las prácticas.

- Reflexionar sobre las competencias del educador social en el ámbito en qué desarrolla las prácticas.

- Enmarcar el proceso de prácticas en un proceso de investigaciónacción.

- Enmarcar el trabajo de prácticas y la información de las prácticas.

- Situar las prácticas en el contexto institucional y orientar su análisis.

- Acompañar la integración del alumnado en el centro de prácticas y reflexionar sobre su relación con éste, el equipo y las personas que acompaña.

- Situar los procesos de acción educativa que se hacen a los centros en un marco teórico que permita analizarlos y comprenderlos.

- Acompañar los procesos de diseño, implementación y evaluación de propuestas educativas. 
Las metodologías utilizadas en los seminarios son diversas. Todas tienen por finalidad dinamizar del espacio de encuentro grupal que permita trabajar los diferentes contenidos creando un espacio de aprendizaje significativo. Para ello es necesario promover dinámicas y metodologías participativas. La responsabilidad debe recaer en el grupo, que se va autogestionando. Así, se contribuirá a crear un sentimiento de pertenencia al grupo de trabajo en qué será posible plasmar aquello que se vive, la experiencia que se va construyendo y las competencias que se van poniendo en juego, individualmente y como referente para los compañeros de grupo.

Los seminarios, en la actualidad, son una matiz de pensamiento para el estudiante en ellos se potencian sus competencias de autonomía profesional, formativa y creativa. Cada seminario toma formas diferentes pero comparte un marco de función docente que hace que el estudiante pase de ser el centro del aprendizaje a impulsar el aprendizaje como un agente formativo más. Son muy valorados por los estudiantes y por los propios tutores-centro. En definitiva, los seminarios favorecen la construcción de la identidad profesional del estudiante y por ello son significativos para todos los implicados.

\section{Tejiendo la corresponsabilidad docente}

Durante mucho tiempo, en nuestro practicum, se ha hablado de la supervisión centrándonos en la figura del tutor-universitario e invisibilizando en esta tarea, inconscientemente, al tutor-centro. Muestra de ello, es que durante un tiempo nos referíamos al profesional del centro como tutor, y al docente universitario como supervisor. Estableciendo diferencias entre una figura y otra.

Se han puesto muchos esfuerzos en estos años en trabajar la función docente del tutor-universitario, sobretodo para superar esas primeras representaciones de algunos docentes que la visualizaban como una docencia simple, fácil y de poca dedicación. Para pasar a construir, una significación compartida y definir los marcos de la docencia y supervisión fundamentada en unos referentes compartidos. Una vez se avanzó en esta línea, se abrió otro foco de interrogación. La formación del estudiante no depende sólo de la función docente del tutor-universitario, sino que con quien pasa más horas y le permite transferir competencias en las acciones prácticas es el tutorcentro. $Y$ aún que ya trabajábamos para mejorar en este aspecto, lo estábamos haciendo desde un foco poco ajustado. Garantizar la información del plan de estudios y las condiciones del practicum, los espacios de comunicación e intercambio no era suficiente para que el tutor-centro descubriese y asumiese su función de supervisión. Entendemos que sin especificarlo desde la coordinación, los tutores-centro ya desarrollaban de alguna forma esta función pero no tenían el reconocimiento y apoyo por parte de la coordinación ni del tutor-universitario.

De ahí, que en los últimos años, se ha trabajado desde el reconocimiento e impulso de la función docente del profesional del centro. Por devenir importantísima, por ser en definitiva en el ejercicio profesional donde se práctica al cien por cien la transferencia de competencias consiguiendo así un incremento de éstas como resultado de las intencionalidades formativas. El profesional ejerce su función docente 
principalmente desde la supervisión y acompañamiento cuyo objetivo es el desarrollo de una identidad profesional a partir del aprendizaje del rol profesional y de la construcción de conocimiento profesional (Vázquez, C. y Porcel, A., 1995). Según Mertens (1998) la supervisión se centra en dos actividades principales: el ejercicio sistemático de la reflexión en la acción (pensar-actuar-pensar) y la cesión de responsabilidades en la acción profesional. El espacio de supervisión ha de ofrecer unas condiciones de aprendizaje en las que los estudiantes desarrollen actitudes abiertas y flexibles, dialogantes, participativas y negociadoras (Hernández Aristu, 2002). Este espacio facilita la revisión de la acción que el estudiante realiza en su centro de prácticas y la revisión del marco conceptual y emotivo que lo condujo a llevar a cabo una acción de una manera determinada o de otra.

A partir de estudios realizados por la coordinación del practicum de Educación social de la Universidad de Barcelona nos han permitido avanzar hacia un modelo más cercano a la construcción participada del conocimiento donde tutor-centro y tutoruniversidad configuran un equipo docente. Éste comparte la finalidad de impulsar la transferencia de competencias y la construcción de la identidad profesional desde el ejercicio profesional y desde la reflexión "en", "desde" y "para" la acción (Schön, 1992).

La función docente de tutor-centro no se realiza en solitario. Las universidades deben impulsar políticas de fomento de la función docente de los profesionales de los centros de prácticas. No sólo mediante incentivos económicos y/o servicios, sino que principalmente desde el apoyo para el desarrollo de planes de prácticas. Es necesario abrir espacios para reflexionar sobre qué contenidos y qué herramientas metodológicas se han de establecer para desprendernos de un modelo de practicum unidireccional y pasar a un modelo sistémico-constructivo. La corresponsabilidad en la función docente del tutor-centro nos ha de ocupar y preocupar.

La función docente del tutor-centro y del tutor-universitario, en el marco del grado de educación social, comparten las mismas finalidades: a) Ofrecer un espacio de intervención socioeducativa para movilizar los recursos: conocimientos, actitudes, habilidades para funcionar en las diferentes situaciones de este espacio; y b) Adquirir las competencias específicas del perfil profesional del educador social a partir de las competencias desarrolladas en el grado mediante la reflexión "en" y "de" la acción. Pero, no sólo podemos compartir finalidades, el paso hacia delante es compartir el marco de las competencias donde cada uno de los agentes deberá definir su propio plan de acción y formación para alcanzarlo. Las competencias que formamos todos, incluyendo al estudiante como formador, son:

- Actitud exploratoria hacia la institución de prácticas y su contexto.

- Comprensión del contexto del centro de prácticas y de su proyecto educativo.

- Interpretación y significación de documentación de programación, regulación y avaluación.

- Observación y sistematización de la práctica del educador/a social.

- Identificación de las funciones de los profesionales que integran el equipo. 
- Actitud profesional ante la institución y en el desarrollo de las acciones profesionalizadoras

- Diseño e implementación de proyectos y acciones socioeducativas a partir de un análisis de la realidad y una priorización de necesidades.

- Actitud autorreflexiva y autovalorativa sobre las propias capacidades y competencias.

- Avaluar sus intervenciones y relaciones educativas establecidas en el centro.

En los cuadernos, anteriormente citados, desde la voluntad de alcanzar la corresponsabilidad, se ha impulsado potenciar la función docente en el proceso formativo, tanto del tutor-centro como del tutor-universitario. Esta función docente comporta la articulación de múltiples funciones implicadas en la relación educativa con el estudiante que tiene sus especificidades en función del agente. Pero también se proponen funciones formativas que comparten, de las que se destacan:

- Hacer partícipe al estudiante de su proceso formativo introduciendo estrategias que lo pongan en el centro del proceso de la reflexiónconstrucción del conocimiento y que le permitan ser consciente de su evolución.

- Tener disponibilidad y proximidad por abordar los aspectos más personales y profesionales que emergen en las prácticas individuales, y convertir estos aspectos en material de trabajo y en contenidos formativos en los seminarios.

- Promover espacios de diálogo y reflexión conjunta para abordar los contenidos que se dan en la cotidianidad de la práctica profesional.

- Diseñar los espacios de trabajo colectivo para promover el trabajo en red en el seno de los seminarios.

- Fomentar relaciones corresponsables entre los agentes implicados en el proceso formativo (estudiante, tutor-centro y tutoruniversitario) que faciliten el seguimiento $y$ el apoyo en el aprendizaje profesional del estudiante.

La corresponsabilidad docente nos lleva a construir un proyecto formativo colectivo que en definitiva repercute en la calidad formativa de los profesionales de la educación social. Por ello, supone un compromiso firme para los tutores-centro y los tutores-universitarios. 


\section{A modo de conclusión: ¿Qué hemos aprendido en los últimos años?}

Una forma de cerrar esta comunicación es compartiendo aquello aprendido en el camino recorrido. Aquello que a cada paso dado hacia delante, y algunos de los dados hacia atrás, ha configurado una manera de entender y desarrollar el practicum.

- El practicum está en permanente reconstrucción.

- Esta reconstrucción implica la revisión constante del marco conceptual de los principios que definen el practicum, de sus referentes organizativos y de la incidencia en la transferencia de las competencias de los estudiantes.

- Las transformaciones son oportunidades y retos formativos que amplifican la calidad del practicum que implican a todos los agentes y que se fundamenta en la reflexión-acción como herramienta de evaluación.

- La calidad del practicum según la propuesta presentada se fundamenta en:

- Perseguir un modelo de practicum que aspira a un partenariado recíproco.

- Impulsar la función docente de todos los implicados (tutorescentro, tutores-universitario y estudiantes).

- Facilitar encuentros colectivos para el intercambio de experiencias formativas que impulsen la corresponsabilidad docente y repensar conjuntamente el practicum.

- Velar por la calidad de la formación práctica que va más allá de la calidad de los centros de prácticas, fundamentándose en la calidad de la relación educativa entre los tres agentes.

- Centrarse en la transferencia de competencias en las prácticas para la construcción de la identidad profesional pone al estudiante no sólo en el centro del aprendizaje sino también su función autoformativa y formativa. El estudiante como activista de su formación y la de sus compañeros y no sólo como protagonista.

- La coordinación del practicum supera las funciones administrativas, articula decisiones técnicas e impulsa innovaciones docentes. Así como, se responsabiliza de impulsar la función docente del tutor-centro y del estudiante.

- Es muy importante el grado de conciencia que tienen los agentes de su implicación en el practicum. Es decir, requiere de la decisión consciente e intencional del grado de implicación y compromiso que se va a tomar en el proceso. Desde el tutor-centro hasta el estudiante, pasando por el tutoruniversidad, el grado de compromiso que pone en el proceso va condicionar la calidad de éste así como la significatividad de los aprendizajes que se hagan.

- El seminario espacio de construcción teórico-práctica desde la reflexión de la acción, fundamentado en el trabajo interprofesional en red. 
- Innovación y creatividad metodológica en el marco de las transformaciones para la evolución de un modelo corresponsable.

Aprendemos permanentemente, transformamos permanentemente pero paso a paso evolucionamos y sistematizamos las claves del modelo de practicum. Sin olvidar que nunca alcanzamos plenamente la utopia de la calidad entre otras cosas porque redefinimos esta calidad que llega a ser inalcanzable pero que nos permite no parar nunca de caminar y soñar juntos por una formación profesional de mayor calidad.

\section{Referencias bibliográficas}

Alegre Beneria, R. (2000). Reflexiones entorno al papel del profesional/tutor como docente, en Relaciones humanas y nuevas tecnologías. Libro de Comunicaciones y Ponencias de 3er Congreso de Escuelas Universitarias de Trabajo Social, Zaragoza: Editorial Mira.

Amoros, P.; Fortuny, M.; Millar, M.D.; Molina, M.C.; Pastor, C.; y Torrado, M. (1997). El practicum de la Diplomatura d'educació social. TEXT-GUIA. Facultat de Pedagogia. Divisió de Ciències de l’Educació. Edicions Universitat de Barcelona.

Bolívar, A. (2008). El discurso de las competencias en España: educación básica y educación superior. Red U. Revista de Docencia Universitaria, número monográfico 11 "Formación centrada en competencias(II)". Consultado (25/09/2010) en http://www.um.es/ead/Red_U/m2/bolivar.pdf

Elliott, J. (1993). El cambio educativo desde la investigación-acción. Madrid: Morata

Estela, Ma J. y Escartin, Mạ J. (1988). La evaluación de prácticas en una Escuela de Trabajo Social. Revista de Treball Social, 109, 162-171.

Freixa, M.; Novella, A.; Pérez Escoda, N.; Fores, A.; Fuentes, N.; Costa, S. y Caramés, M. (2010). Quadern de pràctiques externes. Material de l'estudiant. Universitat de Barcelona. Barcelona: Facultad de Pedagogía. Universidad de Barcelona (inédito).

Hernández Aristu, J. (2002). La supervisión como sistema de evaluación continua que garantiza la calidad de los servicios. Documentación Social, 128, 219-240.

Kisnerman, N. (1999). Reunión de conjurados (Conversaciones sobre supervisión). Buenos Aires: Editorial Lumen-Humanitas

Lobato, C. (1996). Desarrollo profesional y prácticas/practicum en la Universidad. Bilbao: Universidad del País Vasco.

Mertens, L. (1998). La gestión por competencia laboral en la empresa y la formación profesional. Madrid: Organización de Estados Iberoamericanos.

Monreal, M.C. y Ruiz, E. (2009). La Formación del Profesorado en el marco de la innovación docente universitaria. El caso de la Universidad Pablo de Olavide. Red-U. Revista de Docencia Universitaria. Número 4. Consultado (25/ 06/ 2011) en http://redaberta.usc.es/redu/index.php/REDU/article/view/114/93 
Novella, A.; Fores, A.; Fuentes, N.; Pérez Escoda, N.; Freixa, M.; S. Costa y M. Caramés (2010). Quadern de pràctiques externes. Material del tutor/a de la Universitat de Barcelona. Barcelona: Facultad de Pedagogía. Universidad de Barcelona (inédito).

Novella, A.; Freixa, M. y Pérez N. (2010). La funció docent del tutor-professional en el marc del practicum. Congrés Internacional Docència Universitària i Innovació. “Nous espais de qualitat en l'Educació Superior. Una anàlisi comparada i tendències". Barcelona, 30 de junio y 1-2 de julio.

Novella, A.; Pérez Escoda, N.; Freixa, M.; Caramés, M.; Costa, S; Fores, A. y Fuentes, N. (2010). Quadern de pràctiques externes. Material del tutor/a del centre. Universitat de Barcelona. Barcelona: Facultad de Pedagogía. Universidad de Barcelona (inédito).

Porcel, A. y Vázquez, C. (1995). La supervisión espacio de aprendizaje significativo instrumento para la gestión. Zaragoza: Certeza- Intress. Colección Intress.

Rué, J. y De Corral, I. (2007, Febrero) Significados de la "Formación docente" en las universidades españolas en el marco del EEES.. Red U. Revista de Docencia Universitaria,número $2 . \quad$ Consultado (15/03/2010) en http://www.um.es/ead/Red_U/2/rue_de_corral.pdf

Salinas, D. (2007). EEES y Practicum: ¿Cómo encajar el practicum en el nuevo marco? En Cid et al. (coords.). IX Simposium Internacional sobre pràcticum. Practicum y Prácticas en empresas en la formación universitaria. Buenas Prácticas en el pràcticum. Poio, 27-29 de junio.

Schön, D. (1992). La formación de profesionales reflexivos: hacia un nuevo diseño de la enseñanza y el aprendizaje de las profesiones. Barcelona: Paidós.

Spackman, N. (2001) "Educación para la práctica profesional: socialización profesional". A Terapia Ocupacional. Madrid: Ed. Médica Panamericana

Tejada, J. (2005). El trabajo por competencias en el practicum: cómo organizarlo y cómo evaluarlo. Revista electrónica de Investigación educativa, 7 (2). Consultado (11/10/2008) de http://redie.uabc.mx/vol7no2/contenido-tejada.html

Toledano, LI. (2003). "Formar educadors socials en la pràctica". Em Planella,J; Vilar, J. L'educació social: projectes, perspectives i camins. Barcelona: Edicions Pleniluni.

- (2004). Marc general: elements i espais d' aprenentatge dels practicum. I Trobada de tutors/es de Practicum en els Serveis Socials de l'Ajuntament de Barcelona. "La funció docent als Serveis Socials de l'Ajuntament de Barcelona. Universitat de Barcelona. Barcelona. Mayo del 2004

Úcar, X. (1998). "Presentación" en RIERA, J. (1998) Concepto, formación y profesionalización del educador social, el trabajador social y el pedagogo social (pp. 9-11). Valencia: Nau llibres.

Vázquez, C. y Porcel, A. (1995). La supervisión espacio de aprendizaje significativo instrumento para la gestión. Zaragoza: Editorial Certeza.

Zabalza, M. A (2002). La enseñanza universitaria. El escenario y sus protagonistas. Madrid: Narcea. 
- (2007). Buenas prácticas en el Practicum: bases para su identificación y análisis. En Cid et al. (Coords.). IX Simposium Internacional sobre practicum. Practicum y Prácticas en empresas en la formación universitaria. Buenas Prácticas en el practicum. Poio, 27-29 de junio.

- (2009). Practicum y formación: ¿En qué puede formar el practicum? En Raposo, M. et al. (Coords.). X Symposium internacional sobre practicum y prácticas en empresas en la formación universitaria, Poio, 29 junio-1 julio de 2009.

- (2011). El practicum en la formación universitaria: Estado de la cuestión. Revista de Educación, 354, 21-43

Cita del artículo:

Novella, A. (2011). Practicum en re-construcción: Tejiendo colectivamente un modelo de prácticas desde la innovación. Revista de Docencia Universitaria. REDU. 9 (3), 259 - 280. Recuperado el (fecha de consulta) en http://redaberta.usc.es/redu

\section{Información acerca de la autora}

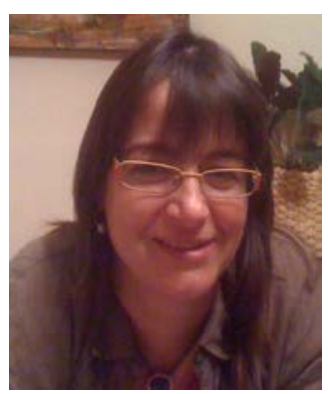

\section{Ana M. Novella Cámara} Universitat de Barcelona

Departamento deTeoría e Historia de la Educación anovella@ub.edu

Doctora en Filosofía y Ciencias de la Educación y Licenciada en Pedagogía por la Universidad de Barcelona. Profesora del Departamento de Teoría e Historia de la Educación de la Facultad de Pedagogía. Integrante del equipo de coordinación de practicum de los estudios de Educación Social. Los proyectos de innovación docente en que participa están vinculados a la calidad de la formación práctica. A nivel de investigación es miembro del GREM, Grupo de Investigación en Educación Moral. Sus principales líneas de trabajo son la participación social de la infancia, la educación para la ciudadanía y la formación cívica. 